\title{
SOBRE EL TRATAMIENTO DE GALIUM L. (RUBIACEAE) EN FLORA IBERICA
}

\author{
Ana ORTEGA-OLIVENCIA y Juan Antonio DEVESA
}

RESUMEN. Sobre el tratamiento de Galium L. (Rubiaceae) en Flora Iberica. El estudio taxonómico del género Galium para Flora Iberica ha revelado la existencia en el territorio de 51 especies y 14 subespecies. Éstas han sido agrupadas en 10 secciones, previamente descritas, reconociéndose para algunas de ellas nuevas series: Galium sect. Galium ser. Trichogaliopsis (Lange) Ortega-Olivencia \& Devesa, Galium sect. Galium ser. Andalusiana Ortega-Olivencia \& Devesa, Galium sect. Platygalium ser. Platygalium (DC.) Ortega-Olivencia \& Devesa, Galium sect. Platygalium ser. Ephedrogalium Ortega-Olivencia \& Devesa y Galium sect. Leiogalium ser. Pulchra Ortega-Olivencia \& Devesa. La serie Ephedrogalium comprende sólo G. ephedroides Willk., taxón para el de se ha estudiado la anatomía foliar, al objeto de evidenciar el número de nervios que poseen sus hojas -tres- y que justifica su inclusión en la sección. Para Galium idubedae (Pau ex Debeaux) Pau ex Ehrend. (Galium sect. Leptogalium Lange) se describe una nueva variedad: Galium idubedae var. humile C. Vicioso ex Ortega-Olivencia \& Devesa.

Palabras clave. Flora Iberica, Galium, Rubiaceae, taxonomía.

SUMMARY. Treatment of Galium L. (Rubiaceae) in Flora Iberica. A taxonomic study of the genus Galium has shown the presence of 51 species and 14 subspecies in Flora Iberica. These taxa are gathered in 10 previously known sections, and in some of them new series are recognized: Galium sect. Galium ser. Trichogaliopsis (Lange) Ortega-Olivencia \& Devesa, Galium sect. Galium ser. Andalusiana Ortega-Olivencia \& Devesa, Galium sect. Platygalium ser. Platygalium (DC.) OrtegaOlivencia \& Devesa, Galium sect. Platygalium ser. Ephedrogalium Ortega-Olivencia \& Devesa and Galium sect. Leiogalium ser. Pulchra Ortega-Olivencia \& Devesa. Ser. Ephedrogalium only comprises G. ephedroides, a species where a study of the foliar anatomy has been carried. Its leaves show three nerves and that character justifies its inclusion into section Platygalium. Also, a new variety is described in G. idubedae (Pau ex Debeaux) Pau ex Ehrend. (Galium sect. Leptogalium Lange): Galium idubedae var. humile C. Vicioso ex Ortega-Olivencia \& Devesa

Key words. Flora Iberica, Galium, Rubiaceae, taxonomy.

El género Galium L. (Rubiaceae) se incluye dentro de la subfamilia Rubioideae y la tribu Rubieae, grupo considerado monofilético (Ehrendorfer, Manen \& Natali,
1994; Manen, Natali \& Ehrendorfer, 1994; Natali, Manen \& Ehrendorfer, 1995). Comprende unas 400 especies (entre $600 \mathrm{y}$ 700, de acuerdo con Phillips, 1951),

Este trabajo ha sido realizado gracias a la financiación del Ministerio de Ciencia y Tecnología a través de los proyectos Flora Iberica V y VI (PB96-0447 y REN2002-04634-C05-04, respectivamente). 
distribuidas por todo el mundo pero con una importante representación en las regiones templadas.

La circunscripción del género ha sido cuestionada sobre la base de estudios filogenéticos efectuados con marcadores moleculares (Manen \& al., 1994); Natali \& al., 1995), que apuntan su posible caracter polifilético. De acuerdo con estos estudios (loc. cit.), (1) los taxones de la sección Aparinoides (p.ej., G. palustre y $G$. elongatum) integran un clado junto con los taxones de Asperula sect. Glabella (p.ej., A. laevigata y $A$. tinctoria), (2) en un mismo clado, igualmente, aparecen reunidos miembros de la sección Platygalium y de los géneros Cruciata y Valantia, y (3) un clado integrado por las especies de Galium sect. Galium, incluye tanto secciones con taxones perennes (Galium, Leiogalium, Leptogalium, Hylaea) como anuales (Kolgyda). Ehrendorfer \& al. (1996), en un estudio utilizando técnicas isoenzimáticas y centrado exclusivamente en las secciones Leptogalium y Leiogalium, demuestran que ambas secciones son dos ramas independientes dentro del clado de Galium sect. Galium.

Estos resultados apuntan el caracter parafilético de los géneros Galium y Asperula (Bremer \& Manen, 2000), si bien todavía son necesarios muchos más estudios ya que buena parte de los efectuados lo han sido con un reducido número de especies. Por esta razón, en el tratamiento del género Galium efectuado para Flora Iberica se ha seguido un criterio práctico, en el que las secciones y series están basadas en características morfológicas, de acuerdo con el tratamiento tradicional (Ehrendorfer \& al., 1976).

Los tratamientos taxonómicos más exhaustivos del género para la Península Ibérica se deben a Lange (1868) y, más recientemente, a Ehrendorfer \& al. (1976) y Krendl (1979, en Galium glaucum aggr.). A
Ehrendorfer, especialista en el género a nivel mundial, se deben numerosos taxones ibéricos nuevos (p.ej., Ehrendorfer, 1960) así como combinaciones de interés (Ehrendorfer, 1974, 1975b; Ehrendorfer \& Krendl, 1974, 1975) y, desde luego, el tratamiento de las secciones y series actualmente reconocidas en Galium (Ehrendorfer, 1975a).

La revisión del género llevada a cabo por los autores con vistas al volumen XV de Flora Iberica, ha entrañado la descripción de tres nuevas especies endémicas (Galium moralesianum y G. talaveranum, OrtegaOlivencia \& Devesa, 2003a; G. belizianum, Ortega-Olivencia, Devesa \& RodríguezRiaño, 2004), así como la publicación de nuevas combinaciones a nivel infraespecífico (Ortega-Olivencia \& Devesa, 2003b). Fruto de esta síntesis, pendiente de publicación, es el reconocimiento para el territorio de 59 especies y subespecies (52 especies y 7 subespecies), así como de numerosas variedades.

La clasificación seguida en el género ha sido la de Ehrendorfer (1975a), reuniéndose los taxones reconocidos en 10 secciones: Galium, Platygalium (DC.) W.D.J. Koch, Hylaea (Griseb.) Ehrend., Trachygalium K. Schum. in Engl. \& Prantl, Aparinoides (Jord.) Gren. in Gren. \& Godr., Leiogalium (DC.) Ledeb., Orientigalium Ehrend., Leptogalium Lange in Willk. \& Lange, Jubogalium Ehrend. y Kolgyda Dumort. Todas incluyen especies perennes, a excepción de las dos últimas, con 1 y 8 especies (con 2 subespecies) anuales, respectivamente.

No obstante, para la correcta agrupación de las especies se ha hecho necesario reconocer algunas series nuevas en Galium sect. Galium, en Galium sect. Platygalium y en Galium sect. Leiogalium (DC.) Ledeb. que se describen en el esquema taxonómico expuesto más abajo, así como una nueva 
variedad para una de ellas (G. idubedae var. humile C. Vicioso ex Ortega-Olivencia \& Devesa).

GALIUM Tourn. ex L., $S p . P l .: 105$ (1753) [Gen. Pl., ed. 5: 46 (1754)], nom. cons.

\section{Sect. 1. Galium}

Galium Ser. Eugalium DC., Prodr. 4: 593 (1830), nom. inval.

Galium Sect. Eugalium (DC.) W.D.J. Koch, Syn. Fl. Germ. Helv.: 332 (1837-1838), nom. inval.

Galium Sect. Galiotypus Dumort., Fl. Belg.: 56 (1827) [Tipo: G. verum L.; Ehrendorfer, 1975a: 3]

Galium [\$] Xanthogalia DC., Prodr. 4: 603

(1830) [Tipo: G. verum L.; Ehrendorfer, 1975a: 4]

Galium Sect. Xanthogalium (DC.) Ledeb., Fl. Ross. 2: 414 (1844)

Galium [\$] Trichogalia DC., Prodr. 4: 599 (1830) [Tipo: G. maritimum L.; K. Schumann in Engler \& Prantl, 1891: 151]

Galium Sect. Trichogalium (DC.) Ledeb., Fl. Ross. 2: 411 (1844)

Tipo: G. verum L. [Phillips, 1951]

La sección comprende sufrútices o hierbas perennes, estoloníferas o rizomatosas, con menor frecuencia cespitosas o pulviniformes, glabras o variadamente pelosas, por lo general sin aculéolos. Las hojas, uninervadas, se disponen en verticilos de 4-10(12), y pueden ser glabras o variadamente pelosas, rara vez escabriúsculas, o bien escábridas, con fuertes aculéolos antrorsos en la haz. Las flores son hermafroditas, sésiles, subsésiles o pediceladas, y presentan una corola tubuloso-infundibuliforme, infundibuliforme, subcampanulada, rotácea, amarillenta, verdosa, rojiza o blanquecina. Los mericarpos son oblongoideos, ovoideos o subesféricos, glabros y con superficie \pm rugosa o papilosa, o bien puberulentos, pubescentes, hirsutos o vilosos, siempre sin pelos uncinados o uncinulados.

Dentro de ella se admiten aquí tres grupos de especies -reconocidos como series- sobre la base de su hábito (sufruticosas, hierbas perennes o bien cespitosas y pulvinulares), tipo de corola (tubuloso-infundibuliforme, subcampanulada, subrotácea o rotácea) y la ausencia o presencia de glándulas en los órganos foliares.

En la serie Galium se reúnen $G$. maritimum L., G. verum L. y G. tunetanum Lam., que se caracterizan por presentar corolas típicamente rotáceas e inflorescencias, aunque polimorfas, no glomerulares, y flores con pedicelos conspicuos.

Un segundo grupo de especies [Galium boissieranum (Steud.) Ehrend. \& Krendl, G. moralesianum Ortega-Olivencia \& Devesa, G. baeticum (Rouy) Ehrend. \& Krendl y $G$. concatenatum Coss.], integran la serie Trichogaliopsis (Lange) Ortega-Olivencia \& Devesa, caracterizadas sobre todo por sus corolas tubuloso-infundibuliformes o subcampanuladas (similares a las de las especies de Asperula), todas ellas además con inflorescencias parciales glomerulares, y flores sésiles o subsésiles.

Finalmente, dos especies ( $G$. erythrorrhizon Boiss. \& Reut. y $G$. pulvinatum Boiss.) integran la serie Andalusiana Ortega-Olivencia \& Devesa. En ellas es característico el hábito cespitoso y pulvinular, la posesión de inflorescencias cimosas simples o poco divididas, paucifloras, con flores pediceladas y corola rotácea $\mathrm{y}$, sobre todo, la presencia de glándulas epidérmicas hinchadas y alargadas, a veces parduscas o anaranjadas, localizadas hacia la parte superior del envés foliar, un carácter que sólo comparten 
también los representantes de la sección Platygalium (DC.) W.D.J. Koch.

\section{Serie 1. Galium}

Galium Sect. Galium Ser. Vera Pobed. in Novosti Sist. Vyssh. Rast. 7: 278 (1971) [Tipo: G. verum L.]

Especies representadas: G. maritimum L., G. verum L. y G. tunetanum Lam.

Serie 2. Trichogaliopsis (Lange) OrtegaOlivencia \& Devesa, comb. \& stat. nov. Asperula Sect. Trichogaliopsis Lange in Willk. \& Lange, Prodr. Fl. Hispan. 2: 304 (1868) [Tipo: Asperula effusa Boiss., = G. boissieranum (Steud.) Enrend. \& Krendl; Ehrendorfer, 1975a: 4]

Tipo: G. boissieranum (Steud.) Enrend. \& Krendl

Especies representadas: Galium boissieranum (Steud.) Ehrend. \& Krendl, G. moralesianum Ortega-Olivencia \& Devesa, G. baeticum (Rouy) Ehrend. \& Krendl y $G$. concatenatum Coss.

Serie 3. Andalusiana Ortega-Olivencia \& Devesa, ser. nov.

Herbaceae, perennes, caespitosopulviniformes. Folia bracteaeque glandulis epidermicis instructa, longis atque prope apicem dilatatis, hyalinis vel tandem aurantiacis aut fuscatis. Inflorescencia equidem cymosa, vel simples atque terminalis, 2-3 flora, vel composita, pauciflora. Flores pedicellati. Corolla rotata, lobulis acutis, nonnumquam apiculatis. Mericarpia simpliciter oblonga vel subovoidea, glabra, rugoso-papillosa.

Hierbas perennes, cespitosopulviniformes. Envés de las hojas y brácteas con glándulas epidérmicas largas e hinchadas cerca del ápice, transparentes, a veces anaranjadas o parduscas, sobre todo en las hojas viejas. Inflorescencia una cima terminal simple, con 2-3 flores, o una cima compuesta pauciflora. Flores pediceladas. Corola rotácea, con 4 lóbulos agudos, a veces apiculados. Mericarpos oblongoideos o subovoideos, glabros, con superficie rugosopapilosa.

Tipo: G. erythrorrhizon Boiss. \& Reut. Especies representadas: G. erythrorrhizon Boiss. \& Reut. y G. pulvinatum Boiss.

Sect. 2. Platygalium (DC.) W.D.J. Koch, Syn. Fl. Germ. Helv.: 331 (1837-1838)

Galium [\$] Platygalia DC., Prodr. 4: 598 (1830) [Tipo: G. rubioides L.; Ehrendorfer, 1975a: 3]

Galium Sect. Cymogalia Pobed. in Schischk., Fl. URSS 23: 712 (1958)], p.p. [Tipo: G. paradoxum Maxim.; Pobedimova in Schischkin, 1958: 712]

Galium Sect. Cymogaliae Ser. Rotundifolia Pobed. in Novosti Sist. Vyssh. Rast. 7: 276 (1971)

Galium Sect. Platygalium Ser. Borealia Pobed. in Novosti Sist. Vyssh. Rast. 7: 276 (1971)

Tipo: G. rubioides L. [Ehrendorfer, 1975a: 3]

Reúne hierbas perennes y sufrútices, estoloníferas, glabras, glabrescentes, escabriúsculas o pelosas. Las hojas, que son trinervadas -a veces de manera imperceptible-, aparecen en verticilos de 4, están provistas de glándulas epidérmicas hinchadas hacia la parte superior del envés y poseen márgenes glabros, antrorso-pelosos o antrorso-escabriúsculos. Las flores, que son hermafroditas y pediceladas, presentan una corola rotácea o subinfundibuliforme, blanquecina o amarillenta. Los mericarpos son oblongoideos, globosos o subreniformes, bien setoso-uncinados, escabriúsculos o 
híspidos, o bien glabros y con superficie rugosa o papilosa.

En el ámbito de la Flora Iberica esta sección comprende 5 especies: $G$. rotundifolium L., G. scabrum L., $G$. broterianum Boiss. \& Reut., G. boreale L. y $G$. ephedroides Willk., todas ellas con órganos foliares en verticilos de 4 y provistos de glándulas epidérmicas hinchadas cerca del ápice de la cara abaxial, carácter este último que también poseen los representantes de Galium sect. Galium ser. Andalusiana.

Las cuatro primeras son hierbas perennes, estoloníferas, con órganos foliares provistos de tres nervios, un carácter exclusivo y que las diferencia de las restantes especies del género. Junto con ellas se incluye $G$. ephedroides que, sin embargo, tiene un biotipo efedroide, y hojas estrechas, prontamente caedizas y en las que apenas si puede reconocerse nervio alguno. Su inclusión en el grupo -aunque en una serie independiente- queda justificada tras el análisis histológico de las hojas (véase Anexo 1), que puso de manifiesto la existencia de un nervio principal, algo ensanchado, y 2 (3-4) nervios más o menos paralelos al mismo desde la base, aunque de menor grosor. Las hojas fueron incluidas en FAA durante c. $36 \mathrm{~h}$ en el caso del material de herbario y c. $24 \mathrm{~h}$ si procedían de plantas en cultivo con el fin de decolorarlas. Posteriormente, se tiñeron con safranina durante $1 \mathrm{~h}$ de cara a la tinción del sistema nerval $y$, finalmente fueron lavadas alternativamente con alcohol al 70\% y FAA, hasta que eran decoloradas al máximo posible. Dichos análisis también se llevaron a cabo en hojas de $G$. boreale de cara a su comparación. De igual forma, en ambas especies se estudió la existencia de glándulas epidérmicas en la cara abaxial de las hojas.

Serie 1. Platygalium (DC.) Ortega-Olivencia \& Devesa, comb. nov.
Galium [\$] Platygalia DC., Prodr. 4: 598 (1830) [Tipo: G. rubioides L.; Ehrendorfer, 1975a: 3]

Tipo: G. rubioides L.

Especies representadas: G. rotundifolium L., G. scabrum L., G. broterianum Boiss. \& Reut. y G. boreale L.

Serie 2. Ephedrogalium Ortega-Olivencia \& Devesa, ser. nov.

Suffruticosae, habitu ephedroideo, nodis inferioribus radicantae, glabrae. Folia obscure trinervia, cito cadentia, glabra, rare nervo centrali atque marginalibus antrorse scabriuscula. Corolla rotata, alba. Mericarpia \pm oblonga, glabra, papillosa.

Sufruticosas, con biotipo efedroide, enraizantes en los nudos inferiores, glabras. Hojas inconspicuamente trinervadas, prontamente caedizas, glabras, rara vez con margen y nervio principal antrorsoescabriúsculo. Flores con corola rotácea, blanca. Mericarpos oblongoideos, glabros y con superficie papilosa.

Tipo: G. ephedroides Willk.

Especies representadas: G. ephedroides Willk.

Sect. 3. Hylaea (Griseb.) Ehrend. in Bot. J. Linn. Soc. 70(1): 3 (1975)

Asperula Sect. Hylaea Griseb., Spic. Fl.

Rumel. 2: 167 (1846)

Asperula [\$] Galioideae DC., Prodr. 4: 585

(1830) [Tipo: Asperula odorata L., =

Galium odoratum (L.) Scop.;

Ehrendorfer, 1975a: 3]

Asperula Sect. Galioideae (DC.) Gren. \&

Godr., Fl. France 2: 47 (1850)

Asperula Sect. Chlorostemma Lange in Willk. \& Lange, Prodr. Fl. Hispan. 2: 304 (1868) [Tipo: Asperula odorata L.,

= Galium odoratum (L.) Scop.; Ehrendorfer, 1975a: 3] 
Galium Sect. Osmogalium Ehrend. in Janch., Cat. Fl. Austriae: 568 (1958), sine descr.

Asperula sect. Trichocarpae Pobed. in Schischk., Fl. URSS 23: 694, 217 (1958) [Tipo: Asperula odorata L., = Galium odoratum (L.) Scop.; Pobedimova in Schischkin, 1.c.]

Galium Sect. Trichocarpa (Pobed.) Pobed. in Novosti Sist. Vyssh. Rast. 7: 275 (1971) [Tipo: G. odoratum (L.) Scop.; Ehrendorfer, 1975a: 3]

Tipo: Galium odoratum (L.) Scop., = Asperula odorata L.) [Ehrendorfer, 1975a: 3]

Reúne hierbas rizomatosas, frecuentemente olorosas, con hojas medias uninervadas y dispuestas en verticilos de 69(10), glabras, excepto en el nervio principal del envés y de los márgenes que son antrorsoescábridos. Las flores son hermafroditas y pediceladas, la corola infundibuliforme o campanulada, blanca, y los mericarpos globosos, con pelos uncinados.

Especies representadas: Galium odoratum (L.) Scop.

Sect. 4. Trachygalium K. Schum. in Engl. \& Prantl, Nat. Pflanzenfam. 4(4): 151 (1891) Galium Sect. Leptogalium Ser. Uliginosa Pobed. in Novosti Sist. Vyssh. Rast. 7: 278 (1971) [Tipo: G. uliginosum L., Pobedimova, 1.c.]

Tipo: G. uliginosum L. [Ehrendorfer, 1975a: 3]

Comprende hierbas perennes, estoloníferas, por lo general trepadoras, con tallos de entrenudos fuertemente retrorsoescábridos en los ángulos y glabros ( $G$. uliginosum y $G$. talaveranum) o hírtulos ( $G$. viridiflorum) en las caras. Las hojas son uninervadas y aparecen dispuestas en verticilos de 5-9, con haz generalmente brillante, glabra, antrorso-escábrida o antrorso-pubescente y envés glabro o hirtopubescente o lanoso, poseyendo el margen una fila de acúleos fuertes y retrorsos, a veces acompañado de 1-varias filas submarginales de acúleos antrorsos.

Las flores son hermafroditas y pediceladas, y presentan una corola crateriforme o subcrateriforme ( $G$. uliginosum) o bien rotácea ( $G$. talaveranum y $G$. viridiflorum), blanca (las dos primeras especies), verdosa o amarillenta (la tercera especie). Los mericarpos son subreniformes, oblongoideos u ovoideos, glabros y con superficie granulosa (G. uliginosum), lisa o algo rugosa ( $G$. talaveranum), o bien hispídulos ( $G$. viridiflorum).

Especies representadas: G. uliginosum L., G. talaveranum Ortega-Olivencia \& Devesa y G. viridiflorum Boiss. \& Reut.

Sect. 5. Aparinoides (Jord.) Gren. in Gren. \& Godr., Fl. France 2: 15, 39 (1850)

Galium [groupe] Aparinoides Jord., Observ. Pl. Nouv. 3: 168 (1846)

Galium Sect. Hydrogalium Ehrend. in Janch., Cat. Fl. Austriae: 568 (1958), sine descr.

Galium Sect. Trachygalium Ser. Palustria Pobed. in Novosti Sist. Vyssh. Rast. 7: 277 (1971) [Tipo: G. palustre L., Pobedimova., 1.c.]

Tipo: G. palustre L. [Ehrendorfer, 1975a: 3]

La sección la integran aquí 2 especies, enraizantes en los nudos inferiores del tallo, con claras apetencias por los lugares húmedos o encharcados. Son plantas que a menudo ennegrecen por desecación y cuyos tallos presentan entrenudos glabros o retrorso-escabriúsculos en los ángulos, con acúleos delicados. 
Las hojas, que son uninervadas y se disponen en verticilos de 4-6, son glabras o a veces con zonas submarginales antrorsoescabriúsculas en la haz y con márgenes antrorso y/o retrorso-escabriúsculos. Las flores son hermafroditas y pediceladas, con corola subrotácea o subcampanulada, blanca o a veces externamete rosada $(G$. debile). Los mericarpos son subreniformes, globosos $\mathrm{u}$ ovoideos, glabros y con superficie granulosa o papiloso-granulosa ( $G$. debile), o bien papilosa o rugosa (G. palustre).

Especies representadas: G. debile Desv. y G. palustre L. (incluye G. elongatum C. Presl, como variedad).

Sect. 6. Leiogalium (DC.) Ledeb., Fl. Ross. 2: 406 (1844)

Galium [\$] Leiogalia DC., Prodr. 4: 593 (1830)

Galium Sect. Hylora Griseb., Spic. Fl. Rumel. 2: 156 (1846) [Tipo: G. longifolium (Sibth. \& Sm.) Griseb.; Ehrendorfer, 1975a: 4]

Tipo: G. sylvaticum L. [Ehrendorfer, 1975a: 4]

Reúne hierbas perennes, menos frecuentemente sufrútices o lignificadas en la base, rizomatosas o estoloníferas, rara vez sin estolones, verdes o glaucas y pruinosas, a menudo con pequeños y brillantes cristales aplanados. Los tallos son glabros o, más rara vez, pubérulos, cortamente tomentosos, papiliformes o hirsutos, sobre todo en la parte inferior, y carecen siempre de acúleos retrorsos.

Las hojas son uninervadas y se disponen en verticilos de (4)6-10(12), presentando a veces el nervio muy ensanchado, y márgenes siempre antrorso-escábridos o antrorsoescabriúsculos, con acúleos casi paralelos a dichos márgenes, excepcionalmente lisos. Las flores son hermafroditas y pediceladas, con corola rotácea o subrotácea, crateriforme o campanulada, blanca, rara vez con tonalidades rosadas y, menos frecuentemente, amarilla o verdoso-amarillenta. Los mericarpos son oblongoideos o subreniformes, glabros, con superficie lisa o algo rugosa, pruinosos o no, muy rara vez con pelos dispersos.

La sección comprende en la flora de Europa 49 especies y subespecies (Ehrendorfer \& al., 1976), tradicionalmente segregadas en 3 series: Erecta Pobed. (28 especies y subespecies), Octonaria (Klokov) Ehrend. (9 especies) y Nemoralia M. Popov. ex Ehrend. (12 especies), pero a las que hay que adicionar la serie Pulchra OrtegaOlivencia \& Devesa, que se reconoce aquí como nueva.

La serie Erecta reúne hierbas o pequeños sufrútices de color verde o glauco, nunca pruinosas, con hojas glabras, rara vez hirsutas, de márgenes antrorso-escábridos o antrorso-escabriúsculos, rara vez lisos. Las flores aparecen sobre pedicelos delgados o gruesos, nunca capilares; la corola es rotácea, blanca, amarilla o verdoso-amarillenta, rara vez con tonalidades rosadas. El ovario posee superficie lisa, finamente papilosa o diminutamente granulosa.

En la serie Pulchra se incluyen hierbas estoloníferas, glabras o glabrescentes, glaucas y pruinosas, con hojas de márgenes lisos o antrorso-escabriúsculos. Las flores, como en la serie anterior, tienen pedicelos delgados o gruesos, nunca capilares, la corola es rotácea, blanca (G. friedrichii) o amarilla (G. belizianum) y el ovario es liso, $\mathrm{y}$ a veces pruinoso.

La serie Octonaria comprende hierbas rizomatosas, glabras, glaucas y pruinosas, con hojas de margen antrorso-escabriúsculo. Las flores aparecen sobre pedicelos delgados, mas no capilares, y presentan una corola típicamente crateriforme, blanca. El ovario es liso y por lo general pruinoso. 
Finalmente, en la serie Nemoralia se reúnen hierbas estoloníferas, glabras, verdes o más o menos glaucas y pruinosas, con hojas de márgenes antrorso-escabriúsculos, flores con pedicelos capilares y corola rotácea $\mathrm{o}$ subrotácea, blanca, y ovario liso y pruinoso, al menos en botón floral.

I. Serie Erecta Pobed. in Novosti Sist. Vyssh. Rast. 7: 279 (1971)

Galium Sect. Brachygalium Rouy in Rouy

\& Camus, Fl. France 8: 11 (1903)

Tipo: Galium mollugo L. [Pobedimova, 1971: 279]

Especies y subespecies representadas: $G$. arenarium Loisel., G. mollugo L. subsp. mollugo, G. mollugo subsp. erectum Syme, G. lucidum All. subsp. lucidum, G. lucidum subsp. fruticescens (Cav.) O. Bolòs \& Vigo, G. lucidum subsp. corrudifolium (Vill.) Bonnier y G. crespianum J.J. Rodr.

II. Serie Pulchra Ortega-Olivencia \& Devesa, ser. nov.

Herbae perennes, stoloniferae, glabrae aut glabrescentes, glaucae atque pruinosae, omnes prope, exsiccatae, haud nigrescentes. Folia in verticillis (4)6-8(9), margine laevia aut antrorse scabridulae. Pedicelli tenues vel incrassati, sed numquam revera capillares. Corolla rotata, glabra, alba aut flava, lobulis acutis vel apiculatis. Ovarium glabrum, laeve, nonnumquam pruinosum.

Hierbas perennes, estoloníferas, glabras o glabrescentes, glaucas y pruinosas, en general no ennegrecidas por desecación. Hojas (4)6-8(9), con margen liso o antrorsoescabriúsculo. Pedicelos delgados o gruesos, nunca capilares. Corola rotácea, glabra, blanca o amarilla, con lóbulos agudos o cortamente apiculados. Ovario glabro, con superficie lisa, a veces pruinoso.
Tipo: G. belizianum Ortega-Olivencia, Devesa \& Rodr. Riaño

Especies representadas: G. friedrichii N. Torres, L. Sáez, Mus \& Rosselló y $G$. belizianum Ortega-Olivencia, Devesa \& Rodr. Riaño

III. Serie Octonaria (Klokov) Pobed. in Novosti Sist. Vyssh. Rast. 7: 278 (1971)

Asperula Ser. Octonariae Klokov in Bot. Mater. Gerb. Bot. Inst. Komarova Akad. Nauk S.S.S.R. 18: 226-227 (1957)]. [Tipo: Asperula octonaria Klokov, = Galium octonarium (Klokov) Pobed.; Klokov, 1957: 227]

Asperula [\$] Galioideae DC., Prodr. 4: 585 (1830) [Tipo: Asperula galioides M. Bieb.; Ehrendorfer, 1975a: 4]

Galium Sect. Asperulopsis Gren. in Gren. \& Godr., Fl. France 2: 14, 18 (1850) [Tipo: Galium glaucum L.; [Ehrendorfer, 1975a: 4]

Asperula Sect. Eugaliopsis Lange in Willk. \& Lange, Prodr. Fl. Hispan. 2: 303 (1868) [Tipo: Asperula galioides M. Bieb.; Ehrendorfer, 1975a: 4]

Galium Sect. Galioides (DC.) Pobed. in Novosti Sist. Vyssh. Rast. 7: 277 (1971) [Tipo: Asperula galioides M. Bieb.; Ehrendorfer, 1975a: 4]

Galium Sect. Asperuloides Pobed., Fl. Part. Eur. URSS 3: 110 (1978), p.p. [Tipo: G. galioides (M. Bieb.) Soó, = $G$. biebersteinii Ehrend.; Pobedimova, 1978: 110]

Tipo: G. octonarium (Klok.) Pobed. (= Asperula octonaria Klokov) [Pobedimova, 1971: 278]

Especies y subespecies representadas: $G$. glaucum L. subsp. australe Franco, $G$. glaucum subsp. murcicum (Boiss. \& Reut.) Bolòs \& Vigo y G. pruinosum Boiss. 
IV. Serie Nemoralia Popov. ex Ehrend. in Bot. J. Linn. Soc. 70(1): 4-5 (1975)

Tipo: Galium sylvaticum L. [Ehrendorfer, 1975a: 5]

Especies representadas: G. laevigatum L.

Observaciones: Bolòs \& Vigo (1996) indican también para el NE de España $G$. sylvaticum L. subsp. sylvaticum y $G$. sylvaticum subsp. aristatum (L.) Rouy \& Camus, basándose en citas de autores antiguos. Los dos taxones, junto con $G$. laevigatum L. y G. schultesii Vest (del C y SE de Europa), forman un grupo de taxones muy relacionados, de difícil diferenciación. Todo el material peninsular estudiado del grupo ha sido adscrito a $G$. laevigatum, que al igual que $G$. schultesii posee estolones.

Sect. 7. Orientigalium Ehrend. in Oesterr. Bot. Z. 98: 435 (1951)

Galium Sect. Leiogalia Ser. Hyrcanica

Pobed. in Novosti Sist. Vyssh. Rast. 7: 279 (1971) [Tipo: G. grusinum Trautv.; Pobedimova, 1971: 279]

Tipo: G. subvelutinum (DC.) C. Koch [Ehrendorfer, 1951: 435; 1975a: 5]

Se incluyen aquí plantas cespitosas y pulvinulares, a veces de aspecto muscinal, con estolones y/o rizomas, glabras o glabrescentes, ennegrecidas tras la desecación ( $G$. cometherhizon) o pajizas (G. pyrenaicum).

Los tallos presentan entrenudos glabros, y carecen de acúleos retrorsos, y las hojas, uninervadas y glabras, aparecen dispuestas en verticilos de 4-8(9) y presentan margen liso, a veces superiormente blanquecino o engrosado y blanco-escarioso. Las flores son hermafroditas y aparecen sobre pedicelos mazudos y carnosos ( $G$. cometherhizon) o no (G. pyrenaicum), con corola rotácea o subrotácea, con lóbulos obtusos o subagudos, blanca, rosada o amarillenta. Los mericarpos, anchamente oblongoideos, son glabros y con superficie lisa o finamente granulosopapilosa.

Especies representadas: G. cometherhizon Lapeyr. y G. pyrenaicum Gouan.

Sect. 8. Leptogalium Lange in Willk. \& Lange, Prodr. Fl. Hispan. 2: 308, 316 (1868) Galium Sect. Leptogalium Ser. Hercynica Pobedimova. in Novosti Sist. Vyssh. Rast. 7: 278 (1971) [Tyро: G. hercynicum Weigel, = G. saxatile L.; Pobedimova, 1.c.]

Tipo: G. pumilum Murray, = G. sylvestre Pollich [Ehrendorfer, 1960: 407]

La sección, sin duda la más complicada del género, comprende hierbas perennes, a veces cespitosas y pulvinulares (p.ej., $G$. cespitosum), rara vez lignificadas en la base, estoloníferas o no, verdes, a veces pajizas o ennegrecidas cuando secas, rara vez glaucas. Sus tallos presentan entrenudos glabros y lisos, o bien son retrorso-aculeados, papiliformes, hirsutos o hirsuto-tomentosos. Las hojas, uninervadas, se disponen en verticilos de 4-9(11), presentan a veces el nervio engrosado (p.ej., G. brockmannii) y margen al menos parcialmente retrorsoescábrido, retrorso-escabriúsculo o papiliforme, a menudo con varias filas submarginales antrorso-escabriúsculas, más rara vez liso (G. idubedae).

Los ejes de la inflorescencia son frecuentemente pubescentes en la base; las flores, hermafroditas y pediceladas, poseen corola rotácea, con lóbulos agudos, rara vez obtusos, sin apículo, rosada, rosado-rojiza, blanca o amarillenta, a veces teñida de rosa. Los mericarpos son oblongoideos o subreniformes, glabros, y su superficie papilosa (la mayoría de las especies) o 
granulosa (G. saxatile).

Especies y subespecies representadas: $G$. cespitosum Lam., G. saxatile L., $G$. balearicum Briq., G. valentinum Lange, $G$. rosellum (Boiss.) Boiss. \& Reut., $G$. papillosum Lapeyr. subsp. papillosum, $G$. papillosum subsp. helodes (Hoffmanns. \& Link) Malag. ex Ortega-Olivencia \& Devesa, G. estebani Sennen, G. marchandii Roem. \& Schult., G. nevadense Boiss. \& Reut., G. pusillum L. subsp. hypnoides (DC.) Arcang., G. brockmannii Briq. y G. idubedae (Pau ex Debeaux) Pau ex Ehrend.

Observaciones: Para Galium idubedae (Pau ex Debeaux) Pau ex Ehrend. in Sitzungsber. Kaiserl. Akad. Wiss., Math.Naturwiss. Cl., Abt. 1, 169: 412-413 (1960), endemismo del C y E de España (Sistema Ibérico y Sierra de Guadarrama), que es relativamente frecuente en claros de matorral y bosques perennifolios o caducifolios, roquedos y pedregales, en sustrato calizo o yesoso, se distinguen dos variedades en función del tamaño de las plantas, de los entrenudos del tallo y las hojas:

a) var. idubedae

Esta variedad se distribuye por las sierras de Espadán, Pina y Peñagolosa, Montes Universales, Sierra de Herrera y Sierra de Guadarrama [Esp.: Cs Cu Gu M $\mathrm{Sg}$ Te Z] e incluye plantas (7,5)11-30 cm de altura, con entrenudos basales y medios menores o iguales que las hojas -los superiores hasta 3,5 veces mayores-, hojas 5-15(23) mm, lineares, por lo general patentes o erecto-patentes.

b) var. humile C. Vicioso, var. nov.

G. idubedae f. humilis C. Vicioso in Bol. Soc. Aragonesa Ci. Nat. 10: 102 (1911), nom. nud.
G. idubedae subsp. humile f. pseudopyrenaicum Rivas Goday \& Borja in Anales Inst. Bot. Cavanilles 19: 472 (1961)

G. idubedae subsp. humile (C. Vicioso) Ehrend., nom. in sched.

G. pusillum f. leioclados C. Vicioso \& F. Beltrán, nom. in sched. (MA 117737)

G. pumillum var. brevipedicellatum Pau, nom. in sched. (MA 117743)

G. micranthum Pau, nom. in sched. (MA 118434)

G. caespitosum subsp. gudaricum Rivas Goday, nom. in sched. (MAF 77064)

G. hypnoides sensu auct. hisp., non Vill. (1787)

Diagnosis: Planta herbacea, 2-9(12) cm alta, internodiis brevioribus quam foliis -vel, si nonnumquam elongata, non ultra 1,5-plo longiora-, foliis 2,7-10(12) mm longis, erectis vel erecto-patentibus, filiformibus.

Holótipo: B. et C. Vicioso Herbarium Aragonense/ CALATAYUD (España) / Galium Idubedae PAU. / $\mathrm{f}^{\mathrm{a}}$ humilis. / In montanis frequens, Sierra de Vicort, / Calatayud, 10 Jul. 1910. Leg. Vicioso, C." (MA 117703)

Esta variedad reúne plantas 2-9(12) cm de altura, con entrenudos menores que las hojas -los superiores, si mayores, no más de 1,5 veces-, hojas 2,7-10(12) mm, filiformes, erectas o erecto-patentes y se localiza en el Sistema Ibérico y la Sierra de Guadarrama [Esp.: (Gu) M Sg So Te V Z]. Aunque en ambas variedades las plantas son glabras y sus hojas presentan márgenes lisos, de manera excepcional pueden encontrarse individuos hirsuto-pelosos a nivel de hojas y entrenudos, con pelos $0,1-0,3 \mathrm{~mm}$, o bien retrorso-escabriœsculos.

Sect. 9. Jubogalium Ehrend. in Oesterr. Bot. 
Z. 105: 212-213 (1958)

Tipo: G. graecum L. [Ehrendorfer, 1958: 213]

Reúne plantas anuales, gráciles, que no ennegrecen cuando secas. Los tallos presentan entrenudos glabros o muy dispersamente escábridos, y las hojas, uninervadas, aparecen en verticilos de (5)6$8(10)$, mostrando haz generalmente ciliada hacia la zona inferior y márgenes antrorsoescabriúsculos. Las flores son hermafroditas y pediceladas, y la corola rotácea, con lóbulos aparentemente obtusos, violácea o purpúrea, a veces verdoso-pardusca. Los mericarpos son subreniformes y presentan la zona comisural glandulosa, la extracomisural con pelos uncinulados.

Especies representadas: G. setaceum Lam.

Sect. 10. Kolgyda Dumort., Fl. Belg.: 55 (1827)

Aparine Moench, Methodus: 640 (1794)

Galium Ser. Aparine (Moench) DC., Prodr. 4: 605 (1830)

Galium Ser. Aparine [\$] Euaparines (Moench) DC., Prodr. 4: 607 (1830), nom. inval.

Galium Sect. Euaparine (Moench) Ledeb., Fl. Ross. 2: 419 (1844), nom. inval.

Galium Sect. Aparine (Moench) W.D.J. Koch, Syn. Fl. Germ. Helv.: 330 (18371838), p. max. p.

Aspera Moench, Methodus: 641 (1794)

[Tipo: Asperula nutans Moench, = Galium murale (L.) All.; Ehrendorfer, 1975a: 5]

Galium [\$] Asperae (Moench) DC., Prodr. 4: 610 (1830)

Galium Sect. Aspera (Moench) Ledeb., Fl. Ross. 2: 421 (1844)

Galium Ser. Aparine [\$] Xanthaparines DC., Prodr. 4: 606 (1830) [Tipo: G. viscosum Vahl, = G. campestre Schousboe;
Ehrendorfer, 1975a: 5]

Galium Ser. Aparine [\$] Leiaparines DC., Prodr. 4: 607 (1830) [Tipo: G. tenuissimum M. Bieb.; Ehrendorfer, 1975a: 5]

Galium Sect. Leiaparine (DC.) Ledeb., Fl. Ross. 2: 418 (1844)

Galium Sect. Vaillantioides W.D.J. Koch, Syn. Fl. Germ. Helv.: 329 (1837-1838) [Tipo: G. saccharatum All., $=G$. verrucosum Huds.; Ehrendorfer, 1975a: 5)

Galium Sect. Pseudaparine Lange in Willk. \& Lange, Prodr. Fl. Hispan. 2: 308, 322 (1868) [Tipo: G. viscosum Vahl; Ehrendorfer, 1975a: 5]

Galium Sect. Pseudovaillantia Lange in Willk. \& Lange, Prodr. Fl. Hispan. 2: 309, 326 (1868) [Tipo: G. verticillatum Danth.; Ehrendorfer, 1975a: 5]

Tipo: G. tricorne Stokes p.p., $=G$. tricornutum Dandy [Ehrendorfer, 1975a: 5]

Comprende plantas anuales, algunas trepadoras (p.ej., G. aparine, $G$. tricornutum), verdes o a veces ennegrecidas cuando secas. Los tallos pueden presentar entrenudos glabros y lisos, fuerte o débilmente retrorso-escábridos, papiliformes, retrorso-escabriúsculos o hirsutos, y las hojas, uninervadas, se disponen en verticilos de (2)3-11, mostrando un nervio tenue o engrosado y margen antrorso, retrorso-escábrido o escabriúsculo.

En la mayoría de las especies las flores son hermafroditas, si bien a veces pueden acompañarse de flores masculinas o estériles ( $G$. tricornutum y $G$. verrucosum); son pediceladas y presentan corola rotácea, a veces con lóbulos erectos, agudos, o a menudo incurvos, generalmente sin apículo, blanca a veces algo rosada superiormente, verdosa, amarillenta o incluso rojiza. Los mericarpos son cilíndricos y \pm curvos en la 
madurez (G. murale) o bien elípticos, subreniformes o subglobosos, y su superficie muy variada: lisa, diminutamente granulosa, obtuso o agudo-papilosa (G. tricornutum), mamiliforme ( $G$. verrucosum), o bien heterogénea ( $G$. murale) u homogéneamente recubierta de pelos uncinados (G. aparine), uncinulados ( $G$. parisiense, G. minutulum, G. verticillatum) o a veces rectos, con base tuberculada o no.

Especies y subespecies representadas: $G$. aparine L. subsp. aparine, G. aparine subsp. spurium (L.) Hartman, G. tricornutum Dandy, G. verrucosum Huds., G. viscosum Vahl, G. parisiense L. subsp. parisiense, G. parisiense subsp. divaricatum (Pourr. ex Lam.) Rouy \& Camus, G. minutulum Jord., G. verticillatum Danth. y G. murale (L.) All.

\section{ANEXO 1}

Relación del material estudiado histológicamente para el análisis de la nerviación

\section{G. boreale L.}

ESPAÑA. BURGOS: Frías, río Ebro, junto al puente romano, 30T VN7635, 6.VIII.1990, $A$. Izuzquiza 2364AI, F. Izuzquiza, A. Mara \& al. (MA 505634). Mena, s.f., Clemente (MA 150790). CUENCA: Balneario de Solán de Cabras, 30T WK7482, 21.VII.1978, G. López (MA 436630, 1 \& 2; MA 436604). Sierra del Tremedal, Hoz del Tajo, WK98, 9.VII.1979, G. López (MA 436600). OVIEDO: Siero, entre San Miguel del Marcenado y el cuartel Cabo Noval, 30T TP7611, 7.VII.1993, M.A. Fernández Casado, J.A. Fernández Prieto \& H.S. Nava (MA 547069). SORIA: Covaleda, WM14, cauce del Duero, 28.VII.1984, G. Mateo (MA 436610, 1 \& 2). VITORIA: Olarizu, WN 2742, 31.VIII.1983, J.A. Alejandre (MA 436583).

G. ephedroides Willk.

ESPAÑA. ALMERÍA: Sierra de Alhama,
13.V.1929, E. Gros, Caroli Pau herbarium hispanicum (MA 117921). Río Aguas, entre Turre y Los Gallardos, 1.III.1970, J. Fernández Casas (MA 337472). Entre Carboneras y Mojácar, 14.V.1972, J. Fernández Casas (MA 409514, 1 \& 2). Proximidades de Almería, 30SWF47, 28.V.1989, J. Pizarro, F. Fernández-González \& D. Sánchez Mata, Itinera Geobotánica (MA 538185). Sierra de Gádor, ctra. Aguadulce-Felix, Km 12-13, margen de ctra., 21.IV.2000, A. Ortega (UNEX 30827). Vícar, ctra. de Aguadulce a Félix, por encima del Campo de Golf La Envía, 2.I.2003, A. Ortega \& I. Olivencia (UNEX 30826).

MARRUECOS: Hab. in rupibus calc. littoris rhiphaei, c. Torres de Alcalá (Beni Bu-Frah), 4.VI.1927, Font Quer, Iter Maroccanum n 606 (MA 117926, $1 \&$ 2). Carretera de Ouarzazate, entre Âït-Saoun y Tizi-n-Tiififft, $30^{\circ} 43^{\prime} 02^{\prime}$ 'N, 6037'06"'W, 28.VI.1997, M. Sequeira \& al., 3306MS (MA 594882).

AGRADECIMIENTOS. Nuestro sincero agradecimiento al Dr. M. Laínz, por la traducción de las diagnosis latinas, y a los conservadores de los herbarios BC, COI, GDA-GDAC, JAEN, MA, MAF, MGC, SEV y UNEX.

\section{BIBLIOGRAFÍA}

BOLÒS, O. \& J. VIGO -1996- Rubiàcies. Flora dels Països Catalans 3: 549-590. Barcino. Barcelona.

BREMER, B. \& J.F. MANEN -2000- Phylogeny and classification of the subfamily Rubioideae (Rubiaceae). Pl. Syst. Evol. 225: 43-72.

EHRENDORFER, F. -1951- Rassengliederung, Variabilitätszentren und geographische Merkmalsprogressionen als Ausdruck der raum-zeitlichen Entfaltung des Formenkreises Galium incanum S.S. (Zur Phylogenie der Gattung Galium. II). Oesterr. Bot. Z. 98: 427-490.

EHRENDORFER, F. -1958- Sect. nova JuboGalium (Rubiaceae: Galium), ein alter, aufgesplitterter Sippenkomplex mit Zentrum 
im südöstlichen Mittelmeergebiet. (Zur Phylogenie der Gattung Galium, V.). Oesterr. Bot. Z. 105: 212-228.

EHRENDORFER, F. -1960- Neufassung der Sektion Lepto-Galium Lange und Beschreibung neuer Arten und Kombinationen (Zur Phylogenie der Gattung Galium, VII.). Sitzungsber. Kaiserl. Akad. Wiss., Math.-Naturwiss. Cl., Abt. 1, 169: 407421.

EHRENDORFER, F. -1975a- Infrageneric taxa in European Rubiaceae: Asperula and Galium. Bot. J. Linn. Soc. 70: 2-6.

EHRENDORFER, F. -1975b- Further taxonomic notes on Rubiaceae in Europe. Pl. Syst. Evol. 124: 173-178.

EHRENDORFER, F. \& F. KRENDL -1974Notes on Rubiaceae in Europe. Bot. J. Linn. Soc. 68: 268-272.

EHRENDORFER, F. \& F. KRENDL -1975Rubiaceae. Correction. Bot. J. Linn. Soc. 71: 274.

EHRENDORFER, F., F. KRENDL \& C. PUFF 1976- Galium. In: T.G. TUTIN \& al. (eds). Flora Europaea 4: 14-36. Cambridge University Press. Cambridge.

EHRENDORFER, F., J.F. MANEN \& A. NATALI -1994- CpDNA intergene sequences corroborate restriction site data for reconstructing Rubiaceae phylogeny. Pl. Syst. Evol. 190: 245-248.

EHRENDORFER, F., R. SAMUEL \& W. PINSKER -1996- Enzyme analysis of genetic variation and relationships in diploid and polyploid taxa of Galium (Rubiaceae). $\mathrm{Pl}$. Syst. Evol. 202: 121-135.

KLOKOV, M. -1957- Asperula Series Octonariae. Not. Syst. (Leningrad) 18: 226-227.

KRENDL, F. -1979- Die Arten der Galium glaucum-Gruppe auf der Iberischen Halbinsel und ein Vergleich mit dem Galium cinereum All. Ann. Naturhist. Mus. Wien 82: 291-318.

LANGE, J. -1868- Rubiaceae Juss. In: M. WILLKOMM \& J. LANGE (eds.). Prodromus Florae Hispanicae ... 2: 299-328. E. Escweizerbart'sche Verlagsbuchhandlung. Stuttgartiae.

MANEN, J.F., A. NATALI \& F. EHRENDORFER -1994- Phylogeny of Rubiaceae-Rubieae inferred from the sequence of a cpDNA intergene region. Pl. Syst. Evol. 190: 195-211. NATALI, A., J.F. MANEN \& F. EHRENDORFER -1995- Phylogeny of the RubiaceaeRubioideae, in particular the tribe Rubieae: evidence from a non-coding chloroplast DNA sequence. Ann. Missouri Bot. Gard. 82: 428439.

ORTEGA-OLIVENCIA, A. \& J.A. DEVESA 2003a- Two new species of Galium (Rubiaceae) from the Iberian Peninsula. Bot. J. Linn. Soc. 143: 177-187.

ORTEGA-OLIVENCIA, A. y J.A. DEVESA 2003b- Nuevas combinaciones en el género Galium L. Acta Bot. Malacitana 28: 206-209. ORTEGA-OLIVENCIA, A., J.A. DEVESA \& T. RODRÍGUEZ-RIAÑO -2004- A new Galium species from NW Portugal. Bot. Helv. 114: $1-6$.

PHILLIPS, E.P. -1951- The genera of South African Flowering Plants. Department of Agriculture. $2^{\text {nd }}$ ed. Ciudad del Cabo.

POBEDIMOVA, E.G. -1958- Galium L. In: B.K. SCHISCHKIN (ed.). Flora URSS 23: 287381. Academiae Scientiarum URSS. Mosqua. POBEDIMOVA, E.G. -1971- Taxa nova Florae URSS, 1. Novosti Sist. Vyssh. Rast. 7: 275281.

POBEDIMOVA, E.G. -1978- Rubiaceae Juss. In G.L. MENITSKY (ed.). Flora Evropeiskoi Chasti SSSR 3: 89-116.

SCHUMANN, K. -1891- Galium Tournef. In A. ENGLER \& K. PRANTL (eds.). Nat. Pflanzenfam. 4(4): 149-153.

Aceptado para su publicación en octubre de 2004

Dirección de los autores. Departamento de Biología y Producción Vegetal: Unidad de Botánica. Facultad de Ciencias. Universidad de Extremadura. 06071-Badajoz (España). 